\section{ORIGINAL RESEARCH}

\author{
R.A. Willinsky \\ J. Peltz \\ L. da Costa \\ R. Agid \\ R.I. Farb
}

K.G. terBrugge

\title{
Clinical and Angiographic Follow-up of Ruptured Intracranial Aneurysms Treated with Endovascular Embolization
}

$\mathbf{E}$ ndovascular coil embolization has emerged within the last 2 decades as a safe and effective treatment option for ruptured intracranial aneurysms. The International Subarachnoid Hemorrhage Trial (ISAT) demonstrated better outcomes for ruptured aneurysms treated with coiling vs clipping, with an absolute risk reduction of $7.4 \%$ and a 7 -year survival advantage, ${ }^{1}$ a finding supported by other studies. ${ }^{2,3}$ However, a high percentage of aneurysmal remnants, unknown long-term stability, and potential rehemorrhage are current concerns with this technique. Incomplete treatment has been reported in $15 \%$ to $65 \%$, and recanalization can occur in $14 \%$ to $34 \%$ of aneurysms treated, with rebleeding rates from 1.2 to $2.6 \%{ }^{4-11}$ Currently, only a few long-term studies address these concerns. The purpose of this study was to characterize the longterm clinical and angiographic outcome of ruptured intracranial aneurysms treated by endovascular techniques, focusing on rehemorrhage and changes in aneurysm morphologic features with time.

\section{Received October 2, 2008; accepted after revision December 4.}

From the Division of Neuroradiology (R.A.W., J.P., L.d.C., R.A., R.I.F., K.G.t.B.) Department of Medical Imaging, Toronto Western Hospital, University of Toronto, Toronto, Canada; and Division of Neurosurgery (L.d.C.), Department of Surgery, Sunnybrook Health Sciences Centre, University of Toronto, Toronto, Canada.

Please address correspondence to Robert A. Willinsky, MD, 399 Bathurst St, 3MC-427, Toronto, ON, Canada, M5T 2S8; e-mail: robert.willinsky@uhn.on.ca

Indicates open access to non-subscribers at www.ajnr.org

DOI 10.3174/ajnr.A1488

\section{Materials and Methods}

The Toronto Western Hospital maintains a prospective data base where clinical, radiologic, and therapeutic information on intracranial aneurysms are collected. A retrospective review was performed to obtain clinical and angiographic data on all consecutive patients who presented to our institution with subarachnoid hemorrhage (SAH) from a ruptured intracranial aneurysm and were successfully treated by coiling between May 1994 and April 2008. All patients with aneurysmal SAH in whom endovascular treatment was completed were included. Data base records were checked for accuracy in electronic and/or paper charts. Follow-up information was obtained from chart notes dictated at our multidisciplinary clinic.

This study encompasses 3 different populations of coiled aneurysms, reflecting changes in our practice with time. Initially, endovascular treatment was performed if surgery was considered too dangerous. Then, during the ISAT trial, case patients were randomly assigned to coiling or clipping, and patients not participating in the trial were treated after a multidisciplinary conference involving the cerebrovascular neurosurgeon and the endovascular therapist. After the ISAT results, endovascular treatment became our first choice for suitable aneurysms. Failed coiling attempts (no coil placed in the aneurysmal sac) and planned parent vessel occlusions were excluded. The influence of age, sex, aneurysm size ( $<5 \mathrm{~mm}, 5-15 \mathrm{~mm}, 15-25 \mathrm{~mm},>25 \mathrm{~mm}$ ) and location, and initial angiographic result (complete, neck remnant, and body remnant) with intraprocedural complications, aneurysmal recanalization, need to re-treat, and rebleeding were analyzed with use of the $\chi^{2}$ test, the $t$ test, and the Fisher Exact test (significance defined as a $P$ value $<.05$ ). The SAS System version 9.1.3 (SAS 
Institute, Cary, NC) was used for all analyses. A similar analysis was performed in a subgroup of patients $(n=153)$ with more than 11 months of follow-up to evaluate the long-term results.

\section{Coiling Technique}

All endovascular treatments were performed with the patients under general anesthesia. Activated clotting time was kept between 250 and 300 seconds by systemic anticoagulation with heparin. The types of platinum coils used were Guglielmi detachable coils (GDC; Boston Scientific, Natick, Mass), MicroCoils (Micrus, San Jose, Calif) MicroPlex coils (Microvention, Aliso Viejo, Calif), and Trufill DCS coils (Cordis, Miami Lakes, Fla). Matrix coils (Boston Scientific) and HydroCoils (Microvention) were the predominant coil in 35 and 36 procedures, respectively. Matrix coils were not used after July 2005 after we demonstrated a greater incidence of major recanalization in patients treated with Matrix compared with bare platinum coils, ${ }^{12}$ and HydroCoil use stopped after reports of hydrocephalus were published. ${ }^{13-15}$ The balloon- or stent-assisted technique was used for selected wide-neck aneurysms, with preference given to the use of balloon-assisted technique if possible. Aneurysms were always packed as well as possible, hoping to achieve complete occlusion.

\section{Follow-up}

We initially performed radiologic follow-up using digital subtraction angiography (DSA) with 3D rotational angiography. Later MR angiography (MRA) became our primary follow-up imaging technique. MRA studies used the "autotriggered elliptical centric-ordered" (ATECO) 3D gadolinium-enhanced technique to evaluate for remnants. The ATECO protocol and its usefulness as a surveillance tool for previously coiled aneurysms have been previously described. ${ }^{16,17}$ Two follow-up protocols were used during 14 years. Initially, imaging studies were performed before discharge, at 6 months, and at 18 months after treatment. After 18 months, those patients with completely occluded aneurysms were followed up with imaging (angiogram or MRA) once every 2 years, and those with aneurysm remnants were followed up once a year. In 2005, the protocol was changed to include an additional imaging study 2 to 3 months after treatment. Angiographic outcomes were categorized as 1) complete occlusion, 2) neck remnant, and 3) body remnant. ${ }^{18,19}$

Clinical evaluations were performed in our outpatient brain aneurysm clinic attended by cerebrovascular neurosurgeons and interventional neuroradiologists. Before 1999, outcome was assessed with use of the Glasgow Outcome Scale (GOS), ${ }^{20}$ and in 1999, we added the modified Rankin Scale. ${ }^{21}$ Follow-up clinical examinations were performed at 2 and 6 months and then on a yearly basis. Earlier follow-up was scheduled if changes in the aneurysm morphologic features were noted.

\section{Results}

For the analysis, we divided our cohort into 2 equal groups, each containing a similar number of patients. The first half of our cohort was treated between May 1994 and June 2004 and the second half, between June 2004 and April 2008. In this study, we refer to group 1 as the group of patients treated before June 2004 and group 2 as those treated after June 2004. From 1994 to 2008, endovascular treatment was attempted in 411 patients (453 aneurysms) who presented with ruptured intracranial aneurysms. Twelve patients had 2 aneurysms coiled at the time of rupture, and 1 patient had 3 aneurysms coiled. Treatment was not possible in 34 cases $(8.3 \%)$ that
Table 1: Patient and aneurysm characteristics

\begin{tabular}{lc}
\hline & Number (\%) \\
\hline Mean age (years) \pm SD & $54.8 \pm 15$ \\
Sex: male/female & $119(32) / 258(68)$ \\
Total number of aneurysms treated & 391 \\
Number of aneurysms treated per patient & \\
1 & $364(97)$ \\
2 & $12(3.2)$ \\
3 & $1(0.3)$ \\
Size of aneurysm treated & \\
$<5 \mathrm{~mm}$ & $131(34)$ \\
$5-15 \mathrm{~mm}$ & $243(62)$ \\
$15-25 \mathrm{~mm}$ & $15(3.8)$ \\
$>25 \mathrm{~mm}$ & $2(0.5)$ \\
\hline
\end{tabular}

Table 2: Aneurysm location (391 aneurysms in 377 patients)

\begin{tabular}{lc}
\hline Location & Number (\%) \\
\hline Anterior circulation & $270(69)$ \\
Anterior communicating artery & $131(34)$ \\
Posterior communicating artery & $78(20)$ \\
Parophthalmic artery & $20(5.1)$ \\
Middle cerebral artery & $18(4.6)$ \\
Carotid termination & $10(2.6)$ \\
Anterior choroidal artery & $7(1.8)$ \\
Anterior cerebral artery & $2(0.5)$ \\
Pericallosal artery & $2(0.5)$ \\
Ophthalmic artery & $2(0.5)$ \\
Posterior circulation & $121(31)$ \\
Basilar artery & $85(22)$ \\
Superior cerebellar artery & $14(3.4)$ \\
Posterior inferior cerebellar artery & $12(3.1)$ \\
Vertebral artery & $5(1.3)$ \\
Posterior cerebral artery & $5(1.3)$ \\
\hline
\end{tabular}

\begin{tabular}{lrrrrr}
\hline \multicolumn{5}{l}{ Table 3: Predischarge outcome of $\mathbf{3 7 7}$ patients } \\
\hline \multicolumn{7}{l}{ Glasgow } & Outcome Scale & \\
\hline Hunt and Hess & 1 & 2 & 3 & 4 & 5 \\
I (131) & 4 & 1 & 1 & 5 & 120 \\
II (74) & 4 & 1 & 0 & 4 & 65 \\
III (94) & 8 & 0 & 10 & 14 & 62 \\
IV (70) & 13 & 3 & 13 & 11 & 30 \\
V (8) & 4 & 1 & 0 & 2 & 1 \\
Total (377) & 33 & 6 & 24 & 36 & 278 \\
\hline
\end{tabular}

were excluded from this analysis. Twenty-five failed attempts occurred before June 2004 (12.1\%), and 9 (4.4\%) occurred after June 2004. A total of 391 aneurysms were treated in 377 patients early after the rupture. Patient characteristics are shown in Table 1 and aneurysm locations in Table 2.

Outcome at discharge of 377 patients is shown in Table 3. Good outcome (GOS, 5) was achieved in 278 patients (74\%). Sixty-six patients $(18 \%)$ had moderate disability or a poor clinical outcome, and 33 patients $(8.8 \%)$ died. In good-grade patients (Hunt-Hess I-III), 247 (83\%) had a GOS of 5 and 16 $(5.3 \%)$ died, whereas in poor-grade patients (Hunt-Hess IV and V), good outcome or moderate disability (GOS 4 and 5) was achieved in only 44 (56\%) and 17 (22\%) died.

There were 53 significant procedural complications in 45 patients $(12 \%$; Table 4$)$ that resulted in a poor outcome or death in 11 patients $(2.9 \%)$. Four of these 11 patients had aneurysm perforations, and 5 had a parent-vessel occlusion or distal emboli. An internal carotid dissection occurred in 1 pa- 
Complication Outcome

\begin{tabular}{|c|c|c|c|c|c|}
\hline Complication & No. of Patients $(\%)$ & No Deficit & Transient Morbidity & Permanent Morbidity & Mortality \\
\hline Aneurysm perforation & $23(6.1)$ & 13 & 6 & 1 & 3 \\
\hline Parent vessel occlusion & $15(4.0)$ & 11 & 3 & 1 & 0 \\
\hline Distal emboli & $11(2.9)$ & 4 & 3 & 3 & 1 \\
\hline Mass effect & $1(0.3)$ & 0 & 0 & 1 & 0 \\
\hline Arterial dissection & $2(0.5)$ & 1 & 0 & 0 & 1 \\
\hline Parent vessel rupture & $1(0.3)$ & 0 & 0 & 0 & 1 \\
\hline
\end{tabular}

Table 5: Glasgow Outcome Scale stratified by Hunt and Hess grade at presentation of 292 patients at last follow-up

\begin{tabular}{lrrrrr}
\hline Hunt and & \multicolumn{5}{c}{ Glasgow } \\
\cline { 2 - 6 } Hess & 1 & 2 & 3 & 4 & 5 \\
\hline I (120) & 0 & 1 & 0 & 5 & 114 \\
II (61) & 0 & 0 & 0 & 1 & 60 \\
III (67) & 0 & 0 & 5 & 4 & 58 \\
IV (42) & 0 & 1 & 7 & 6 & 28 \\
V (2) & 0 & 1 & 0 & 0 & 1 \\
Total (292) & 0 & 3 & 12 & 16 & 261 \\
\hline
\end{tabular}

tient, and mass effect from the coil mass causing a permanent third cranial nerve palsy occurred in another. One patient had a rupture of the basilar artery during a balloon-assisted coiling procedure. In the entire cohort, aneurysm perforation occurred in 23 patients $(6.1 \%)$. There were 16 aneurysm perforations $(8.5 \%)$ in group 1 and $7(3.7 \%)$ in group 2 . Aneurysm perforation resulted in transient morbidity or no deficit in 19 $(82.6 \%)$ of 23 patients, permanent morbidity in 1 patient, and death in 3 patients ( 4 poor outcomes of 23 [17.3\%]). Parentvessel occlusions and/or distal emboli occurred in $22(5.8 \%)$ of all patients: 16 patients $(8.5 \%)$ in group 1 , and 6 patients (3.2\%) in group 2. Thromboembolic complications resulted in transient morbidity or no deficit in 17 (77.3\%) of 22 patients, permanent morbidity in $4(18.2 \%)$, and death in 1 $(4.5 \%)$. This patient who died had an associated aneurysm perforation.

Abciximab (ReoPro; Eli Lilly, Indianapolis, Ind) was used in 27 patients for thrombus formation at or near the parent vessel/coil interface. Since August 2002, abciximab has been used in 27 (11\%) of 237 patients. The drug was either given intravenously as a bolus $(25 \mu \mathrm{g} / \mathrm{kg})$ or intra-arterially at a lower dose (4-12 mg titrated to response) through a microcatheter near the intraluminal clot. Our current protocol is to use the full, single intravenous dose, and in only a few cases did we give the 12-hour intravenous infusion following the initial intravenous or intra-arterial bolus. Most patients (25/27) had no neurologic deficits related to thromboembolic complications. One patient experienced permanent neurologic sequelae from the distal emboli, and another had a second hemorrhage from the aneurysm the following day and was retreated with surgical clipping. Technical complications without clinical significance occurred in 8 patients: coil herniation into the parent vessel in 4 patients, coil stretching in 2 patients, stent inadvertently deployed proximal to the aneurysm in 1 patient, and traumatic catheterization of the urethra in 1 patient.

Complete follow-up was achieved in 292 (85\% of surviving) patients. GOS at last follow-up is shown in Table 5. The average interval between treatment and the last follow-up was 22.3 months (range, 20 days to 119.8 months). Additional treatment was required in 31 patients $(11 \%)$. Eleven $(3.7 \%)$ were re-treated with surgical clipping and 21 (7.1\%) underwent additional embolization, with 5 requiring a third embolization. One patient was initially re-treated with coils and subsequent clipping. At their last clinical examination, 245 patients $(84 \%)$ had no clinical deficits. Rehemorrhage after coiling occurred in 8 patients $(2.1 \%)$. Six patients had an early rehemorrhage, defined as less than 30 days' postcoiling (5 within 48 hours of treatment). Outcomes of rehemorrhages were poor (Table 6). Four of the patients who had rebleeding were in group 1, and 3 patients who had rebleeding were in group 2. Complete occlusion at the time of coiling was achieved in 2 of the 6 aneurysms that re-bled early. One patient treated with a combination of Matrix and bare platinum coils had rebleeding at 5.5 months. The rebleeding rate with Matrix $(2.8 \%)$ was similar to the overall results. There was no rebleeding in patients treated with HydroCoil or a combination of HydroCoil and bare platinum.

A total of 166 subjects had follow-up durations longer than 11 months. Information on 153 patients was used to analyze which factors were associated with aneurysm recanalization and the need for re-treatment (13 patients were excluded because of unavailable information regarding aneurysm recanalization). The presence of a body remnant after coiling was significantly related to aneurysm recanalization $(P<.0001)$ and with the need for re-treatment $(P=.0433)$. The very small number of rebleedings ( 2 in this subgroup) did not allow for any statistical correlation.

\section{Imaging Results}

Immediate postcoiling angiography demonstrated complete occlusion of $170(43 \%)$ of the aneurysms, a neck remnant in $142(36 \%)$, and a body remnant in $79(20 \%)$. Follow-up imaging after discharge was available for $276(70.6 \%)$ of the aneurysms (270 patients [71.6\%]). At the time of last follow-up, recanalization was observed in $56(20.3 \%)$ of 276 aneurysms: $26(18.8 \%)$ for group 1 and $30(21.7 \%)$ for group 2 . From the 170 aneurysms with initial complete occlusion, follow-up imaging was obtained in 121 aneurysms (118 patients). Eightyseven aneurysms (71.9\%) remained stable, with no change in their angiographic appearance after initial treatment. Thirtyfour aneurysms (28\%) recanalized, with 17 developing neck remnants and 16 developing body remnants. The average time between treatment and detection of recanalization was 9.6 months (range, 0.1-46 months). Eight (6.6\%) of the 121 were re-treated ( 4 coiled, 4 clipped). Of the 142 aneurysms with neck remnants on initial angiogram, 106 aneurysms (104 pa- 


\begin{tabular}{|c|c|c|c|c|c|c|c|c|}
\hline Age & Sex & Initial $\mathrm{HH}$ & $\begin{array}{c}\text { Location of Treated } \\
\text { Aneurysm }\end{array}$ & Size & $\begin{array}{c}\text { Immediate Angiographic } \\
\text { Result }\end{array}$ & $\begin{array}{l}\text { Time to Rebleeding } \\
\text { (Days) }\end{array}$ & Intervention & GOS \\
\hline 62 & Male & 4 & BA & $14 \mathrm{~mm}$ & Body remnant & 3606 & None & $1^{*}$ \\
\hline 61 & Male & 1 & VA & $6.3 \mathrm{~mm}$ & Neck remnant & 161 & Recoiling & $2^{*}$ \\
\hline 84 & Female & 2 & MCA & $3.0 \mathrm{~mm}$ & Complete occlusion & 29 & None & 1 \\
\hline 59 & Male & 4 & AcomA & $6.0 \mathrm{~mm}$ & Neck remnant & 5 & None & 4 \\
\hline 46 & Female & 1 & MCA & $6.5 \mathrm{~mm}$ & Body remnant & 1 & None & 1 \\
\hline 67 & Female & 2 & AcomA & $5.0 \mathrm{~mm}$ & Complete occlusion & 1 & None & 1 \\
\hline 42 & Male & 5 & Pericallosal & $5.3 \mathrm{~mm}$ & Neck remnant & 1 & Clipping & $4 \dagger$ \\
\hline 70 & Female & 3 & AcomA & $6.1 \mathrm{~mm}$ & Neck remnant & 1 & None & 1 \\
\hline
\end{tabular}

Note:-GOS indicates Glasgow Outcome Scale; HH, Hunt and Hess; BA, basilar artery; VA, vertebral artery; MCA, middle cerebral artery; AcomA, anterior communicating artery.

${ }^{*}$ Recanalization evident on post rehemorrhage angiography.

† Patient given intravenous abciximab.

tients) had follow-up imaging. Fifty aneurysms (47\%) became completely occluded, and $46(43 \%)$ were stable. Ten aneurysms $(9.4 \%)$ developed body remnants. The average time between treatment and detection of recanalization was 6.3 months (range, 2.0-11 months). Ten of the 106 (9.4\%) aneurysms were re-treated: 5 were recoiled once, 3 were recoiled twice, 2 were clipped, and 1 was recoiled and subsequently clipped. Of the 79 aneurysms with body remnants on immediate postcoiling imaging, 49 (in 49 patients) had follow-up imaging. Twenty-two aneurysms (45\%) showed favorable evolution with smaller remnants or complete occlusion, and $15(31 \%)$ were stable. Twelve aneurysms (24\%) showed recanalization with interval enlargement of the body remnant. All 12 were retreated: 6 were recoiled once, 2 were recoiled twice, and 4 were clipped. The average time between treatment and detection of recanalization was 6.7 months (range, 0.2-24.2 months).

\section{Discussion}

The primary goal of treatment of a ruptured aneurysm is to prevent rehemorrhage. However, rehemorrhage after treatment is still an issue. The ISAT trial demonstrated an overall rehemorrhage rate of $4.9 \%$, with an incidence of rebleeding after surgical clipping of $1.2 \% .{ }^{1}$ Rebleeding risk after surgical clipping has been reported to be low, varying from $1.9 \%$ to $3.2 \%$, higher with incomplete sac occlusion. ${ }^{22-25}$ Recanalization is a known issue with endovascular treatment, but its impact on rebleeding rates is not completely clarified. Despite recanalization rates as high as $38 \%$, reports on the incidence of rebleeding after coiling vary. ${ }^{26-30}$ In our study, the overall incidence of rehemorrhage was $2.1 \%$, with time until rebleeding ranging from 0 days to 118.6 months (almost 10 years). Six of the 8 patients had a subsequent hemorrhage within 30 days of treatment, 5 within 48 hours. We found a risk for rebleeding of $1.6 \%$ during the first 30 days after coiling, decreasing to $0.7 \%$ after that. There were 2 episodes of late rebleeding, 1 almost 10 years after treatment, the longest interval between treatment and rebleeding after endovascular embolization reported so far. ${ }^{4,9,11}$ The second rebleed occurred less than 6 months from treatment. This latter rebleed led to changes in our practice regarding follow-up imaging. Ruptured aneurysms treated with coiling are now imaged with MRA before discharge, at 2 to 3 months and then 6 months after treatment for assessment of early recanalization. Follow-up imaging is then performed yearly or every other year, depending on the presence of a remnant.
Outcome after rehemorrhage was poor, with a $62.5 \%$ mortality rate, concurrent with another series reporting high mortality rates after early rerupture. ${ }^{4,8}$ It is interesting to note that rebleeding rates were not different in completely occluded aneurysms compared with aneurysms with neck or body remnant $\left(\chi^{2}, 1.135 ; P=.28\right)$, a result supported by others. Rebleeding occurred after complete occlusion in 2 patients, a neck remnant in 4 , and a body remnant in 2 . Sluzewski et al ${ }^{28}$ also found no significant difference in the risk for rehemorrhage between complete and incomplete occlusion, with 4 of 6 early ( $<30$ days) reruptures occurring after complete occlusion of the aneurysm. Different results were shown by investigators in the Cerebral Aneurysm Rerupture After Treatment study on predictors of rehemorrhage after treatment of ruptured intracranial aneurysms, who found the degree of aneurysm occlusion to be highly predictive of the risk for rerupture, which increased progressively as the packing attenuation decreased. ${ }^{27}$ Accordingly, there was no difference in the re-treatment rates of aneurysms with neck remnant $(9.4 \%)$ compared with completely occluded $(6.7 \%)$ aneurysms $\left(\chi^{2}, 0.776 ; P=\right.$ .38 ). As expected, aneurysms with a body remnant at the end of the coiling were re-treated more often $(24 \%$ re-treatment rate) than lesions completely occluded or left with a neck remnant $\left(\chi^{2}, 5.959 ; P=.0146\right)$. In ruptured aneurysms, we no longer strive for complete occlusion in every case because those with a neck remnant have a similar natural history as those that are completely occluded. We do not struggle for a perfect angiographic result because the complication rate may increase with attempts to achieve the ideal picture.

Periprocedural complications occurred in $12 \%$ of our patients, with permanent morbidity or mortality rate related to the procedure in $2.9 \%$, a complication rate similar to other studies. Our permanent morbidity and mortality rates were lower than other single-center studies on ruptured aneurysms. ${ }^{3,18,30-33}$ This may reflect our conservative strategy regarding the immediate angiographic result. The most common procedural complication was intraprocedural rupture $(6.1 \%)$. This rate is similar to that of reports in the literature. ${ }^{11,18,32,34}$ In our cohort, a small size (defined as an aneurysm sac smaller than $5 \mathrm{~mm}$ ) had no influence in the rate of intraprocedural rupture. The risk for intraprocedural perforation was $7.6 \%$ for aneurysms less than $5 \mathrm{~mm}$ and $5.0 \%$ for aneurysms more than $5 \mathrm{~mm}\left(\chi^{2}, 0.715 ; P=.3979\right)$. Other authors have demonstrated an increased risk for perforation in small aneurysms. Nguyen et a ${ }^{35}$ recently demonstrated that the risk for rupture increases from $2.3 \%$ for aneurysms more 
Table 7: Complication rates in Groups 1 and 2

\begin{tabular}{|c|c|c|c|c|c|c|}
\hline & \multicolumn{3}{|c|}{ Group 1 (1994-June 2004) } & \multicolumn{3}{|c|}{ Group 2 (June 2004-April 2008) } \\
\hline & No. of Patients & Total Patients & $\%$ & No. of Patients & Total Patients & $\%$ \\
\hline $\begin{array}{l}\text { Failed attempts } \\
\text { Intraprocedural complications }\end{array}$ & 25 & 206 & 12.1 & 9 & 205 & $\overline{4.4}$ \\
\hline Aneurysm perforations & 16 & 189 & 8.5 & 7 & 188 & 3.7 \\
\hline Thromboembolic events & 16 & 189 & 8.5 & 6 & 188 & 3.2 \\
\hline Rehemorrhage & 4 & 189 & 2.1 & 3 & 188 & 1.6 \\
\hline Recanalization & 26 & 138 & 18.8 & 30 & 138 & 21.7 \\
\hline
\end{tabular}

Table 8: Long-term follow-up studies after coiling of ruptured intracranial aneurysms

\begin{tabular}{|c|c|c|c|c|c|}
\hline Author & $\begin{array}{l}\text { No. of } \\
\text { Patients }\end{array}$ & $\begin{array}{l}\text { Mean Follow-up } \\
\text { Time in Months } \\
\text { (Longest Follow-up) }\end{array}$ & $\begin{array}{l}\text { Procedural Complications } \\
\text { (Permanent Morbidity } \\
\text { and Mortality) }\end{array}$ & $\begin{array}{c}\text { Recanalization } \\
\text { Rate }\end{array}$ & $\begin{array}{l}\text { Rehemorrhage } \\
\text { (Early/Late) }\end{array}$ \\
\hline Present study, 2008 & 377 & $22.3(120)$ & $12 \%(2.9 \%)$ & $20.3 \%$ & $1.6 \% / 0.5 \%$ \\
\hline Aikawa, $2007^{26}$ & 227 & 50.4 (NS) & NS & $10 \%$ & $\mathrm{NS} / 2.6 \%$ \\
\hline Sluzewski, $2005^{29}$ & 393 & $47.7(120)$ & NS & NS & $\mathrm{NS} / 1.27 \%$ \\
\hline Friedman, $2003^{39}$ & 83 & $19.1(112)$ & $19 \%(2 \%)$ & $15 \%$ & $0 \% / 0 \%$ \\
\hline Sluzewski, $2003^{30}$ & 160 & $37.1(72)$ & NS $(3.8 \%)$ & $25 \%$ & $\mathrm{NS} / 1.2 \%$ \\
\hline Kremer, $2002^{9}$ & 79 & $41(74)$ & NS & NS & $\mathrm{NS} / 3 \%$ \\
\hline Byrne, $1999^{4}$ & 317 & $22.3(65)$ & NS & $14.7 \%$ & $\mathrm{NS} / 1.6 \%$ \\
\hline
\end{tabular}

Note:-NS indicates not studied.

than $3 \mathrm{~mm}$ to $11.7 \%$ for those less than $3 \mathrm{~mm}$. Our size categorization was arbitrary, and the cutoff value for small aneurysms was at $5 \mathrm{~mm}$. The results may have been different if very small lesions ( $<3 \mathrm{~mm}$ in size) were analyzed separately.

Thromboembolic complications occurred in $5.8 \%$ of all patients and were more frequent in the first half of our experience $(8.5 \%$ of patients in group 1 and $3.2 \%$ of patients in group $\left.2\left[\chi^{2}, 3.859 ; P=.0495\right]\right)$. A major difference between the 2 groups is the introduction of abciximab (ReoPro) during endovascular procedures after 2004. Abciximab, a monoclonal antibody glycoprotein IIa/IIIb inhibitor, has shown to be very effective in the treatment of acute thromboembolic complications during endovascular procedures. ${ }^{36}$ We used abciximab (intravenously or intra-arterially) in 27 patients when thrombus was evident during the procedure, and there were no symptomatic thromboembolic events in $93 \%$ of these patients. One patient had an ischemic stroke in the motor cortex, and another had a subsequent hemorrhage from the treated aneurysm the following day and underwent craniotomy and clipping. Intracerebral hemorrhages have been described with abciximab use. ${ }^{37}$

Imaging follow-up (MRA and/or conventional angiography) was achieved in $70.5 \%$ of the treated aneurysms, and similar to other studies, our recanalization rate was $20.2 \% .^{4,5,7,11,18}$ Recanalization occurred regardless of the initial angiographic result, but as expected, the risk was higher if a body remnant was left $\left(\chi^{2}, 11.791 ; P=.0006\right)$. Grunwald et $\mathrm{al}^{7}$ demonstrated that only $12.7 \%$ of completely occluded aneurysms recanalized compared with $40 \%$ of those with initial $80 \%$ to $95 \%$ occlusion, and Raymond et $\mathrm{al}^{11}$ demonstrated recanalization rates of $20.0 \%$ in completely occluded aneurysms vs $40.1 \%$ recanalization of aneurysms with neck remnants and $51.1 \%$ recanalization of aneurysms with body remnants. This difference, however, was significant only in the short term. In our study, the interval between treatment and recanalization was similar for patients with initial neck and body remnants (average,
6.6 months) but slightly longer for completely occluded aneurysms (average, 9.6 months), suggesting that recanalization occurs after complete occlusion, but at a slower pace. Recanalization of a completely occluded aneurysm was detected up to 46 months after treatment.

The overall incidence of complications, including failed attempts and recanalization (Table 7 ), decreased from $14.8 \%$ in group 1 compared with $6.9 \%$ in group $2\left(\chi^{2}, 4.825 ; P=.0280\right)$. This may be attributed to the learning curve of the endovascular therapists, higher volumes of patients in group 2, technical advances, and the use of abciximab. The number of patients is the same in both groups, though group 1 encompasses 10 years and group 2 , only 4 years. This finding is in agreement with that of other studies demonstrating the benefit of surgical treatment of intracranial aneurysms in high-volume centers. ${ }^{38}$ Multisection CTA with 3D reconstructions; rotational angiography with high-quality images; and availability of better microcatheters, coils, stents, and balloons are likely to have had a positive influence in our results.

\section{Study Limitations}

Our study had some limitations. There were $15 \%$ of patients lost to follow-up, and imaging was not available in all. The long study period encompassed a shift in management strategies and multiple technical advances, which made comparison difficult. Follow-up imaging was initially done exclusively with DSA and later shifted to gadolinium-enhanced MRA. This change could have resulted in differences in interpretation of the imaging results, though the high sensitivity of MRA in the detection of aneurysm remnants after coiling was demonstrated by our group. ${ }^{19}$ The modified Rankin Scale, a more sensitive measure of outcome, was not incorporated into our data base until 1999. Despite the limitations, our study was among the largest in single-center studies (Table 8) on the long-term follow-up of endosaccular treatment of ruptured aneurysms. ${ }^{4,9,11,26,29,30,39}$ 


\section{Conclusions}

Long-term clinical and angiographic follow-up after coiling of ruptured aneurysms confirms its efficacy as a primary treatment technique. Rebleeding rates after treatment are low, but recanalization remains an issue, even in aneurysms that are initially completely occluded. Long-term imaging follow-up is advised. Initial angiographic appearance is not a good predictor of rehemorrhage, recanalization, and long-term outcome.

\section{References}

1. Molyneux AJ, Kerr RS, Yu LM, et al. International Subarachnoid Aneurysm Trial (ISAT) of neurosurgical clipping versus endovascular coiling in 2143 patients with ruptured intracranial aneurysms: a randomised comparison of effects on survival, dependency, seizures, rebleeding, subgroups, and aneurysm occlusion. Lancet 2005;366:809-17

2. Flett LM, Chandler CS, Giddings D, et al. Aneurysmal subarachnoid hemorrhage: management strategies and clinical outcomes in a regional neuroscience center. AJNR Am J Neuroradiol 2005;26:367-72

3. Taha MM, Nakahara I, Higashi T, et al. Endovascular embolization vs surgical clipping in treatment of cerebral aneurysms: morbidity and mortality with short-term outcome. Surg Neurol 2006;66:277-84; discussion 284

4. Byrne JV, Sohn MJ, Molyneux AJ, et al. Five-year experience in using coil embolization for ruptured intracranial aneurysms: outcomes and incidence of late rebleeding. J Neurosurg 1999;90:656-63

5. Cognard C, Weill A, Spelle L, et al. Long-term angiographic follow-up of 169 intracranial berry aneurysms occluded with detachable coils. Radiology 1999;212:348-56

6. Gallas S, Pasco A, Cottier JP, et al. A multicenter study of 705 ruptured intracranial aneurysms treated with Guglielmi detachable coils. AJNR Am J Neuroradiol 2005;26:1723-31

7. Grunwald IQ, Papanagiotou P, Struffert T, et al. Recanalization after endovascular treatment of intracerebral aneurysms. Neuroradiology 2007;49:41-47

8. Holmin S, Krings T, Ozanne A, et al. Intradural saccular aneurysms treated by Guglielmi detachable bare coils at a single institution between 1993 and 2005: clinical long-term follow-up for a total of 1810 patient-years in relation to morphological treatment results. Stroke 2008;39:2288-97

9. Kremer C, Groden C, Lammers G, et al. Outcome after endovascular therapy of ruptured intracranial aneurysms: morbidity and impact of rebleeding. $\mathrm{Neu}$ roradiology 2002;44:942-45

10. Pandey AS, Koebbe C, Rosenwasser RH, et al. Endovascular coil embolization of ruptured and unruptured posterior circulation aneurysms: review of a 10year experience. Neurosurgery 2007;60:626-36; discussion 636-37

11. Raymond J, Guilbert F, Weill A, et al. Long-term angiographic recurrences after selective endovascular treatment of aneurysms with detachable coils. Stroke 2003;34:1398-403

12. Kimchi TJ, Willinsky RA, Spears J, et al. Endovascular treatment of intracranial aneurysms with Matrix coils: immediate posttreatment results, clinical outcome and follow-up. Neuroradiology 2007;49:223-29

13. Fanning NF, Willinsky RA, ter Brugge KG. Wall enhancement, edema, and hydrocephalus after endovascular coil occlusion of intradural cerebral aneurysms. J Neurosurg 2008;108:1074-86

14. Kang HS, Han MH, Lee TH, et al. Embolization of intracranial aneurysms with hydrogel-coated coils: result of a Korean multicenter trial. Neurosurgery 2007; 61:51-58; discussion 58-59

15. Mitha AP, Wong JH, Lu JQ, et al. Communicating hydrocephalus after endovascular coiling of unruptured aneurysms: report of 2 cases. J Neurosurg 2008;108:1241-44

16. Farb RI, McGregor C, Kim JK, et al. Intracranial arteriovenous malformations: real-time auto-triggered elliptic centric-ordered 3D gadolinium-enhanced MR angiography-initial assessment. Radiology 2001;220:244-51
17. Farb RI, Nag S, Scott JN, et al. Surveillance of intracranial aneurysms treated with detachable coils: a comparison of MRA techniques. Neuroradiology 2005;47:507-15

18. Roy D, Milot G, Raymond J. Endovascular treatment of unruptured aneurysms. Stroke 2001;32:1998-2004

19. Agid R, Willinsky RA, Lee SK, et al. Characterization of aneurysm remnants after endovascular treatment: contrast-enhanced $M R$ angiography versus catheter digital subtraction angiography. AJNR Am J Neuroradiol 2008;29:1570-74

20. Jennett B, Bond M. Assessment of outcome after severe brain damage. Lancet 1975; 1:480-84

21. Platelet glycoprotein IIb/IIIa receptor blockade and low-dose heparin during percutaneous coronary revascularization. The EPILOG Investigators. N Engl J Med 1997;336:1689-96

22. Wermer MJ, Greebe P, Algra A, et al. Incidence of recurrent subarachnoid hemorrhage after clipping for ruptured intracranial aneurysms. Stroke 2005;36:2394-99

23. David CA, Vishteh AG, Spetzler RF, et al. Late angiographic follow-up review of surgically treated aneurysms. J Neurosurg 1999;91:396-401

24. Feuerberg I, Lindquist C, Lindqvist M, et al. Natural history of postoperative aneurysm rests. J Neurosurg 1987;66:30-34

25. Tsutsumi K, Ueki K, Usui M, et al. Risk of recurrent subarachnoid hemorrhage after complete obliteration of cerebral aneurysms. Stroke 1998;29:2511-13

26. Aikawa H, Kazekawa K, Nagata S, et al. Rebleeding after endovascular embolization of ruptured cerebral aneurysms. Neurol Med Chir (Tokyo) 2007;47: 439-45; discussion 446-47

27. Johnston SC, Dowd CF, Higashida RT, et al. Predictors of rehemorrhage after treatment of ruptured intracranial aneurysms: the Cerebral Aneurysm Rerupture After Treatment (CARAT) study. Stroke 2008;39:120-25

28. Sluzewski M, van Rooij WJ. Early rebleeding after coiling of ruptured cerebral aneurysms: incidence, morbidity, and risk factors. AJNR Am J Neuroradiol 2005;26:1739-43

29. Sluzewski M, van Rooij WJ, Beute GN, et al. Late rebleeding of ruptured intracranial aneurysms treated with detachable coils. AJNR Am J Neuroradiol 2005;26:2542-49

30. Sluzewski M, van Rooij WJ, Rinkel GJ, et al. Endovascular treatment of ruptured intracranial aneurysms with detachable coils: long-term clinical and serial angiographic results. Radiology 2003;227:720-24

31. Byrne JV. Acute endovascular treatment by coil embolisation of ruptured intracranial aneurysms. Ann R Coll Surg Engl 2001;83:253-56; discussion 257

32. Park HK, Horowitz M, Jungreis C, et al. Periprocedural morbidity and mortality associated with endovascular treatment of intracranial aneurysms. AJNR Am J Neuroradiol. 2005;26:506-14

33. Vinuela F, Duckwiler G, Mawad M. Guglielmi detachable coil embolization of acute intracranial aneurysm: perioperative anatomical and clinical outcome in 403 patients. J Neurosurg 1997;86:475-82

34. Deng J, Zhao Z, Gao G. Periprocedural complications associated with endovascular embolisation of intracranial ruptured aneurysms with Matrix coils. Singapore Med J 2007;48:429-33

35. Nguyen TN, Raymond J, Guilbert F, et al. Association of endovascular therapy of very small ruptured aneurysms with higher rates of procedure-related rupture. J Neurosurg 2008;108:1088-92

36. Aviv RI, O'Neill R, Patel MC, et al. Abciximab in patients with ruptured intracranial aneurysms. AJNR Am J Neuroradiol 2005;26:1744-50

37. Qureshi AI, Saad M, Zaidat OO, et al. Intracerebral hemorrhages associated with neurointerventional procedures using a combination of antithrombotic agents including abciximab. Stroke 2002;33:1916-19

38. Solomon RA, Mayer SA, Tarmey JJ. Relationship between the volume of craniotomies for cerebral aneurysm performed at New York state hospitals and in-hospital mortality. Stroke 1996;27:13-17

39. Friedman JA, Nichols DA, Meyer FB, et al. Guglielmi detachable coil treatment of ruptured saccular cerebral aneurysms: retrospective review of a 10-year single-center experience. AJNR Am J Neuroradiol 2003;24:526-33 